\title{
Benefit on daily listening with technological advancements: comparison of basic and premium category hearing aids
}

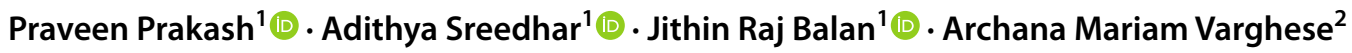

Received: 11 July 2021 / Accepted: 23 December 2021 / Published online: 17 January 2022

(c) The Author(s), under exclusive licence to Springer-Verlag GmbH Germany, part of Springer Nature 2022

\begin{abstract}
Purpose The aim of the study was to compare the user-rated benefit of two categories of hearing aids, mainly the basic and premium categories of hearing aids.

Methods A questionnaire was administered on 102 hearing aids users (47 basic and 55 premium category users) with severity of hearing loss ranging from mild to moderately severe sensorineural hearing loss. The questionnaire administered was divided into mainly seven subscales which included speech intelligibility in quiet and in noise, ease of communication, the efficiency of noise reduction, localization, quality of music perception and cost effectiveness. The effect of duration of daily usage of hearing aids on performance among these different subscales was also assessed.

Results Ease of communication was rated better by premium hearing aid users, whereas the cost effectiveness was rated to be better by basic users. There was no significant difference observed between performances of basic versus premium category of hearing aids in other listening domains assessed. There was no significant difference in any of the listening domains with daily usage duration for both categories of hearing aid users.

Conclusion The users of premium category devices revealed better ease of communication in daily environments, whereas performance of these devices on other listening domains remains questionable. Cost effectiveness was reported to be better by the users of basic hearing aids. A prospective and controlled paired series comparison of hearing aid performance needs to be performed to confirm these findings.
\end{abstract}

Keywords Hearing aid benefits $\cdot$ Basic versus premium hearing aids $\cdot$ Comparison of benefits $\cdot$ Advanced technology

\section{Introduction}

Hearing loss can have a deleterious impact on daily life of an individual that might include increased isolation from family and social life, depression, anxiety, reduced self-efficacy, and impaired overall mental health [1]. Even though hearing

Praveen Prakash

praveenprakashp1849@gmail.com

Adithya Sreedhar

adithyass67@gmail.com

Jithin Raj Balan

jithinrajb@gmail.com

Archana Mariam Varghese

archana.agnes@gmail.com

1 Department of Audiology, All India Institute of Speech and Hearing, Manasagangothri, Mysuru 570 006, India

2 Department of Speech Pathology and Audiology, Amrita Institute of Medical Sciences, Kochi 682041, India aids are considered as one of the best rehabilitation options for most common hearing losses, persons with hearing loss may start using them after a span of at least a few years from the onset of hearing loss. Most individuals go for a hearing aid only when the limitations due to the reduced hearing sensitivity and associated handicap affect daily life seriously, where the amplification of sounds becomes inevitable [1,2]. Unfortunately, almost $75 \%$ of the population who could benefit from hearing aids does not start using hearing aids [3].

The cosmetical concern is presumed to be one of the factors that baffle in choosing the right hearing aids. In a country like India, there are no insurance policies that cover for the cost of hearing aid. Hence, the financial burden also plays a significant role in deciding whether to choose a hearing aid or not. For those who can afford hearing aids, the category of the hearing aid (entry level or advanced level) one may choose, or whether he can go for monaural or binaural fitting may also be influenced by financial concern [4]. 
Latest hearing aid technologies are more promising compared to the older generations in terms of speech perception in noise, noise reduction algorithms, directional hearing, wireless connectivity, geotagging, and automatic program selection based on data logging [5, 6]. Hearing aids with an increased number of channels or more advanced features are expected to have better performance based on noise reduction, speech discrimination, directionality, and other individual-based requirements than hearing aids with basic features. This has been supported by the hearing aid users and the laboratory tests and claimed by the manufacturers $[7,8]$. Similarly, the use of more than one microphone and real-time directional control has been reported to improve horizontal localization [9].

Even though the verification of hearing aid functioning can be carried out through real-ear measurements and functional gain measurements, [10-12] it is also warranted to quantify the post-fitting experiences and obtain realistic feedback and level of satisfaction from the users of hearing aids. This can help streamline the hearing aid prescription methods across professionals and evaluate whether any modifications are required for the successful hearing aid fitting. Moreover, these subjective measures of hearing aid outcome help quantify hearing aid benefits in real-life situations compared to the objective tests.

There are numerous widely used subjective tools that can be utilized to evaluate patient benefit from hearing aid use [13-17]. Many surveys reported users' satisfaction with hearing aid by evaluating factors such as age, duration of use, quality of life, income, unilateral versus bilateral fitting, and degree of hearing loss, [18, 19]. However, these studies have been conducted outside India. To better understand the hearing aid fitting practices, follow-up services and to provide quality services for patients with hearing loss in the Indian scenario, there is a need to study the factors influencing fitting, usage, and satisfaction of hearing aid among users of different categories of hearing aids. Even though it is recommended to follow uniform standards and protocols for hearing aid fitting worldwide [12], Easwar et al. [20] conducted a study on clinical practices performed by audiologists working across India, reporting that only less than $12 \%$ of audiologists performed real-ear measurements. It was also found that less than $30 \%$ of the audiologists performed outcome measures like aided sound field testing or in the form of questionnaires. Hence, conducting a survey to obtain feedback from hearing aid users via questionnaire is a reliable and realistic method to collect the responses from the hearing aid users. It mainly covers the notable difficulties and issues faced by them. The cost of the device plays a vital role among the non-audiological aspects like cosmetic appeal, dexterity, and individualized requirements like smartphone connectivity $[4,18,19]$. With the incorporation of additional features and more convincing designs, the price of hearing aids also increased compared to the basic models. There is a dearth of research comparing hearing aid performance across basic and premium categories of hearing aids. With the outbreak of Coronavirus pandemic in 2019, almost all the sectors of the economy faced an intensive negative impact and thus financial instability has become another concern for every individual [21]. Hence, reducing the financial burden on treatment such as hearing aid fitting is also an important aspect that needs to be addressed. In this connection, the authors aimed to carry out a study that compared the benefit derived from a basic hearing aid and a premium category hearing aids to quantify whether the extra investment done on the premium category of hearing aids are justifiable or not. The study aimed to quantify and compare the benefit of patients who had undergone fitting with basic models of hearing aids (Four- six-channel with basic features, Maximum Retail price $\leq ₹ 30,000$ ) and premium models of hearing aids (Nine-channel or more, additional features like beamformers, speech-seeking directional microphones, data logging, advanced noise reduction technology and multiple microphone ports; with a maximum Retail price $\geq ₹ 65,000$ ) through a subjective rating. The primary objectives of the study are (1) to compare the performance scores reported by users of basic and premium hearing aids, (2) to compare the daily listening domains for monaural and binaural hearing aid users of different category of hearing aids, (3) to compare the effect of overall duration (in months or years) of use of hearing aids on the major listening domains, (4) to compare the effect of daily usage duration (in hours) on each domain irrespective of the hearing aid category.

\section{Method}

A retrospective review of the records of patients who had undergone their hearing aid fitting during last 5 years (between 2015 and 2020) was considered for the study. Hearing aid user details were collected from the patient database from the medical records of All India Institute of Speech and Hearing, Mysuru, India. The duration of the study was 6 months (beginning from December 2020 and ended by May 2021). Demographic details, results of the audiological evaluation such as type and degree of hearing loss, progression of hearing loss, cause of hearing loss, speech perception scores, age of initial hearing aid fitting, model of the hearing aids opted, and the number of hearing aids procured were collected. A total of 152 patients were invited to participate in the study. Those individuals, whose recent audiological evaluation results could not be obtained due to their time constraints were excluded $(n=29)$. Among those assessed through online modalities, incomplete responses due to their personal reason were not considered for the study $(n=20)$. 
One participant was excluded from the study as the reported daily usage duration of the hearing aid was less than $1 \mathrm{~h}$.

\section{Subjects}

A total number of 102 hearing aid users ( 47 basic and 55 premium category users), age ranging from 17 to 55 years (mean age $=44.10$ years, $\mathrm{SD}=7.56$ ) with post-lingual acquired hearing loss were considered for the study. Subjects with minimum usage duration of 6 months since their first fit, having hearing aids in one ear or both ears, were included in the study. The degree of hearing loss included ranged from mild to moderately severe, having flat configuration of hearing loss. To maintain homogeneity, individuals with a bilateral and symmetrical degree of hearing loss were selected for the study. The scores for speech identification in quiet were $75 \%$ or above for all the subjects as per most recent audiological evaluation record. Individuals who had not undergone audiological evaluation during last 6 months were tested as a part of periodic audiological evaluation. Individuals who crossed the age of 60 were excluded considering the age-related neural degeneration that could affect the auditory perception apart from the hearing loss [22].

For all hearing aid users, appropriate counselling was done regarding the care and maintenance of the hearing aids, along with the description of controls. The user manuals along with the developed pamphlets regarding the use of hearing aids were given to each patient. For both basic and advanced hearing aids, a detailed description regarding the use of control was explained to each patient. For hearing aids having direct Bluetooth streaming, the patient's smartphone was connected to the hearing aids, and a demonstration of streaming to phone and accepting the phone calls from the hearing aids was also explained.

All the subjects were the hearing aid users of major international manufacturers like Sonova, Starkey, GN Resound, William Demant Holding, Sivantos, and Widex. The entrylevel and premium category hearing aids were matching with their basic features (e.g., in the number of channels, compression handles, feedback canceller, and for advanced hearing aids, the features such as auto sense, wind noise reduction, wireless connection, binaural communications, etc.). It was made sure that each category of hearing aids across different manufactures were matching almost in the features provided as listed above, and the outliers, if present were excluded. All the hearing aids were set to first fit with NAL-NL2 (the second generation of prescription procedures from The National Acoustic Laboratories) fitting formula, with enabled volume control and the program button activated. For the advanced hearing aids, all other controls were set to manage automatically. Hearing aids which was programmed with other fitting formulae was excluded from the current study. Hence there was no variability to subgroup the hearing aid users except the degree of hearing loss. The questionnaire was administered as a face-to-face interview and also via telephonic based on the patient preference due to the restricted movement by the COVID-19 pandemic.

During the event of hearing aid selection, all those patients who did not had any financial constraints in taking up premium category devices, were given trials with both the basic and premium category of the hearing aids before prescribing the hearing aids. Apart from the routine hearing aid verification procedures that included aided speech identification scores and testing for tolerance levels, the final decision to opt whether he/she required basic or premium device was based on the patient's preference of advanced features and perceived quality and naturalness of speech. However, for those who had financial restrictions to take up premium category hearing aid/s, hearing aid trials was given with different models of basic category hearing aids to choose the best fit for them.

\section{Questionnaire}

A questionnaire consisting of 21 questions, prepared after reviewing various published questionnaires on hearing aid benefits and satisfaction [13-17]. These questions assessed seven subscales which included: (1) speech intelligibility in quiet and in noise, with respect to male/female/child speaker, over the telephone, and listening to Television, (2) ease of communication, (3) efficiency of noise reduction, (4) naturalness of speech, (5) localization (6) music perception and (7) cost effectiveness (Refer to Appendix 1). The questions under each subsection had choices which varied from categories like "poor" to "very clear", "seldom" to "very frequently", and "yes" or "no"; where the options were allocated based on the content of each test item. The scoring for polar questions was 0 and 1 corresponding to 'NO' or 'YES', respectively. For the remaining questions, the responses were scored from 1 to 4 , where 1 indicated the least benefit for that specific question and 4 indicated the highest benefit possible. The range of possible scores for each listening domain and the rating for each option has been depicted in Appendix 1. The following is an example of a question under the subsection 'Speech intelligibility' Q20-How well is the clarity of speech when heard over telephone/mobile?

The questionnaire was prepared in English. It was given to two audiologists who had experience of a minimum 6 years or more to validate the content and for appropriate suggestions. Based on these suggestions and corrections, the final version of the questionnaire was prepared. It was also translated from English to Kannada (a language, which is predominantly used in Karnataka, a southern state in India) by two audiologists who were native Kannada speakers to make the Kannada version and reverse translated to English 
by another audiologist who was fluent in both Kannada and English to avoid the ambiguity in the translation.

\section{Administration of questionnaire}

The questionnaire was administered through a face-to-face interview for the hearing aid users who came for followup evaluations. Some users were reached via telephone, and the questionnaire was administered at their convenience. The clients were explained regarding each domain or subscales mentioned in the questionnaire, and they were asked to choose the most appropriate rating for each question. For those who had difficulty reading or comprehending the test items, the investigators filled the questionnaire based on the responses obtained from the patients through a face-to-face interview. For those who are literate to read the English version of the form and familiar with the digital forms, the questionnaire was shared via Google forms for self-reporting. It was made sure that all participants undergoing assessment through different modes understood the content of each question before rating the response to reduce the variability in the response rating. The responses were analyzed and compared between basic and premium hearing aid users across domains using appropriate statistical tools.

\section{Ethical considerations}

The personal information of all the clients was maintained confidential. The subjects were informed about the objective and procedures to be followed, and informed consent was taken from the participants prior to administering the questionnaire. Before reviewing the records, written permission for accessing medical records from the Institute was obtained. All the procedures followed in the study were in agreement with the 'ethical approval committee of the institute and complied with the Declaration of Helsinki'.

\section{Results}

The present study aimed to compare the hearing aid benefit across the basic and premium categories of hearing aids, divided based on the features and the cost. The response data were analyzed for normal distribution using the Shapiro-Wilk test for normality using Statistical Package for Social Sciences (SPSS, version 21) [23]. It was found that the majority of the data obtained were following a non-normal distribution $(p<0.05)$. Hence, non-parametric tests were carried out for statistical analysis.

The details including number of monaural/binaural users and degree of hearing loss are displayed in Fig. 1. The percentage of participants divided based on the duration

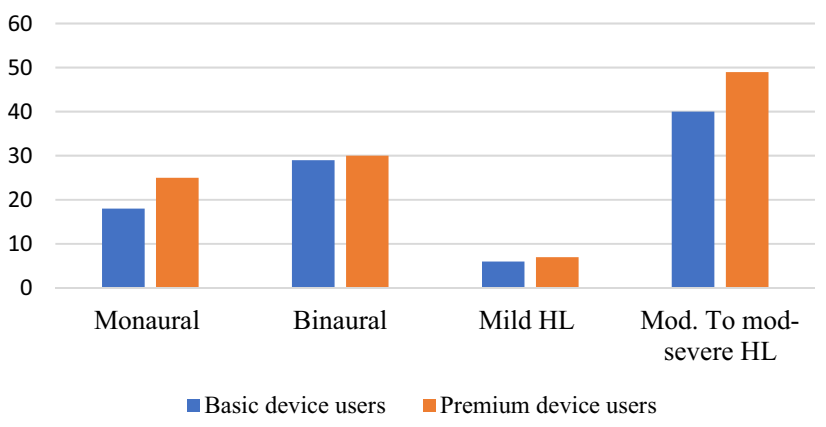

Fig. 1 Number of hearing aid users based on the category of devices and degree of hearing loss

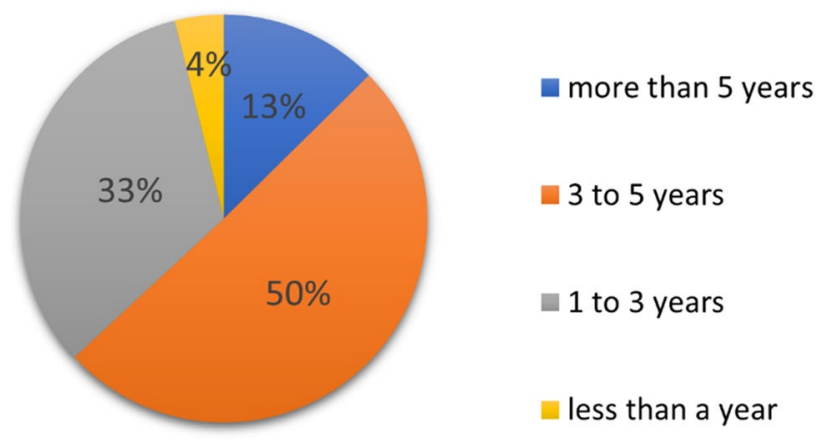

Fig. 2 The percentage of participants divided based on their durations between the onset of hearing loss and the initial fitting of hearing aid

between the onset of hearing loss and initial fitting of the device is displayed in Fig. 2.

\section{Comparison of the performance scores reported by users of basic and premium category hearing aids}

The mean, standard deviations, median and range of the scores obtained from each listening domain among the two categories of hearing aid users are given in Table 1. The scores obtained in each domain for the two categories of hearing aid users were compared using the Mann-Whitney $U$ test. The results revealed no statistically significant differences between the scores of major subscales except for 'ease of communication' and 'cost-effectiveness'. The premium hearing aid users rated better for ease of communication, whereas the cost effectiveness was rated better by basic users. The results are displayed in Table 2 .

Analysis of speech intelligibility in quiet and in noise was separately done for mild hearing loss and moderate to moderately severe hearing loss across the two categories of hearing aid users. Among individuals with mild hearing loss, there was no significant difference in the scores obtained across the two categories of the hearing aids for the 
perception of speech in quiet $[U=22.5, Z=-0.28, p>0.05]$ and also for speech perception in noise $[U=20, Z=-0.64$, $p>0.05]$. Similarly, among moderate to moderately severe hearing loss, there was no significant difference in the scores obtained across the two categories of the hearing aids for the perception of speech in quiet $[U=754, Z=-2.12, p>0.05]$ and also for the perception of speech in noise $[U=817$, $Z=-1.43, p>0.05]$.

\section{Comparison of performance in each listening domain among monaural and binaural hearing aid users}

Among the monaural and the binaural hearing aid users, the responses obtained in each domain were compared between the basic and premium hearing aid users. The mean scores, standard deviation, median, and range of the scores obtained by monaural and binaural hearing aid users across different domains are mentioned in Tables 3 and 4, respectively. Since all the participants had bilateral symmetrical hearing loss, improvements in localization abilities were compared among binaural hearing aid users only across two categories of hearing aids. For both monaural and binaural hearing aid users, the Mann-Whitney $U$ test showed no significant difference between the performance of basic and premium hearing aids across all domains $(p>0.05)$.

\section{Comparison of scores obtained across each domain based on years of hearing aid use}

The effect of duration of hearing aid usage on the mean scores obtained in the following seven listening domains is shown in Fig. 2. The scores obtained in all domains were compared using the Kruskal-Wallis test. The result revealed no statistically significant difference for different duration of hearing aid usage on the listening domains assessed $(p>0.05)$.
Table 1 Mean, Standard deviation (S.D), median, and range of scores obtained by both categories of hearing aid users across each domain

Table 2 Results of MannWhitney $U$ test comparing the scores of two categories of hearing aid users across all domains

\begin{tabular}{|c|c|c|c|c|c|c|c|c|}
\hline & \multicolumn{4}{|c|}{ Entry-level hearing aid users $(N=47)$} & \multicolumn{4}{|c|}{$\begin{array}{l}\text { Higher-end hearing aid users } \\
(N=55)\end{array}$} \\
\hline & Mean & SD & Median & Range & Mean & SD & Median & Range \\
\hline Speech Intelligibility & 12.6 & 2.41 & 13 & $6-16$ & 13.25 & 2.48 & 14 & $6-17$ \\
\hline Ease of communication & 8.47 & 2.27 & 9 & $2-14$ & 9.61 & 2.45 & 10 & $4-14$ \\
\hline Efficiency of noise reduction & 3.98 & 1.18 & 4 & $0-5$ & 3.82 & 1.14 & 4 & $0-5$ \\
\hline Naturalness of speech & 2.02 & 0.53 & 2 & $1-3$ & 2.07 & 0.5 & 2 & $1-3$ \\
\hline Quality of music perception & 2.57 & 0.54 & 3 & $1-3$ & 2.75 & 0.67 & 3 & $1-4$ \\
\hline Cost effectiveness & 4.6 & 0.61 & 5 & $3-5$ & 3.63 & 1.12 & 4 & $0-5$ \\
\hline
\end{tabular}

\begin{tabular}{llllll}
$\begin{array}{l}\text { Speech } \\
\text { Intelligibil- } \\
\text { ity }\end{array}$ & $\begin{array}{l}\text { Ease of com- } \\
\text { munication }\end{array}$ & $\begin{array}{l}\text { Efficiency of } \\
\text { noise reduction }\end{array}$ & $\begin{array}{l}\text { Naturalness } \\
\text { of speech }\end{array}$ & Music perception & Cost effectiveness \\
\hline-1.534 & -2.629 & -0.663 & -0.495 & -1.893 & -5.065 \\
$>0.05$ & $<0.01^{*}$ & $>0.05$ & $>0.05$ & $>0.05$ & $<0.01^{*}$ \\
\hline
\end{tabular}

*Indicates the significant level at $1 \%$ level

Table 3 Mean, standard deviation, median and range values of monaural hearing aid users across different domains for the two categories of hearing aids

\begin{tabular}{|c|c|c|c|c|c|c|c|c|}
\hline & \multicolumn{4}{|c|}{ Entry-level $(n=18)$} & \multicolumn{4}{|c|}{ Higher-end $(n=25)$} \\
\hline & Mean & Std. deviation & Median & Range & Mean & Std. deviation & Median & Range \\
\hline Speech intelligibility scores & 12 & 3.06 & 13 & $6-16$ & 13.54 & 2.37 & 14 & $9-17$ \\
\hline Ease of communication & 8.71 & 2.54 & 9 & $4-14$ & 10.04 & 2.25 & 10.5 & $6-14$ \\
\hline Efficiency of noise reduction & 4.29 & 0.92 & 5 & $2-5$ & 3.52 & 1.45 & 4 & $0-5$ \\
\hline Naturalness of speech & 1.82 & 0.64 & 2 & $1-3$ & 2.08 & 0.40 & 2 & $1-3$ \\
\hline Quality of music perception & 2.41 & 0.62 & 2 & $1-3$ & 2.88 & 0.73 & 3 & $1-4$ \\
\hline
\end{tabular}




\section{Comparison of the effect of everyday usage duration (in hours) on the scores obtained in each listening domain for the entry level and premium category of hearing aids}

Mean, standard deviation, median, and range of the scores obtained by users with different duration of everyday usage of entry-level and premium categories are displayed in Tables 5 and 6 , respectively.

The effect of usage duration (in hrs) across domains irrespective of the category of the device was assessed using the Kruskal-Wallis test. Results revealed no significant differences with daily usage duration in all the listening domains for both categories of hearing aid users $(p>0.05)$.

It was also noted that among the 55 users of premium hearing aids, $<5 \%$ used wireless connectivity technologies. On interviewing, it was realized that a majority of the users were not using the Bluetooth feature available in the hearing aids due to a lack of technological knowledge.

\section{Discussion}

In the current study, we compared the rating provided by hearing aid users across various listening domains. Comparing the benefit of basic versus the premium models across these domains showed no differences existed in their overall scores rated by the users except for ease of communication. In the premium hearing aids, many features other than the volume control are set to function automatically. The individuals using premium models reported better scores compared to basic hearing aid users for ease of communication. As per the questionnaire (refer to Appendix 1), these individuals required fewer instances of change in programs

Table 4 Mean, standard deviation, median and range of the scores obtained across domains among binaural hearing aid users for the two categories of hearing aids

\begin{tabular}{|c|c|c|c|c|c|c|c|c|}
\hline & \multicolumn{4}{|c|}{ Entry-level $(n=29)$} & \multicolumn{4}{|c|}{ Higher-end $(n=30)$} \\
\hline & Mean & Std. deviation & Median & Range & Mean & Std. deviation & Median & Range \\
\hline Speech intelligibility scores & 12.97 & 1.955 & 13 & $8-16$ & 13 & 2.586 & 14 & $4-17$ \\
\hline Ease of communication & 8.31 & 2.173 & 9 & $2-12$ & 9.23 & 2.596 & 10 & $4-13$ \\
\hline Efficiency of noise reduction & 3.76 & 1.3 & 4 & $0-5$ & 4.1 & 0.96 & 4 & $2-5$ \\
\hline Naturalness of speech & 2.14 & 0.441 & 2 & $1-3$ & 2.07 & 0.583 & 2 & $1-3$ \\
\hline Improvement in Localization & 2.41 & 0.628 & 2 & $1-3$ & 2.36 & 0.678 & 2 & $1-3$ \\
\hline Quality of music perception & 2.64 & 0.488 & 3 & $2-3$ & 2.63 & 0.615 & 3 & $1-3$ \\
\hline
\end{tabular}

Table 5 Mean, standard deviation (SD), median and range of scores obtained by entry-level users across domains based on daily usage duration

\begin{tabular}{|c|c|c|c|c|c|c|c|c|c|c|c|c|}
\hline \multirow[t]{2}{*}{ Daily usage duration } & \multicolumn{4}{|c|}{ Less than $5 \mathrm{~h}(n=1)$} & \multicolumn{4}{|c|}{$5-10 \mathrm{~h}(n=11)$} & \multicolumn{4}{|c|}{ More than $10 \mathrm{~h}(n=35)$} \\
\hline & Mean & SD & Median & Range & Mean & $\mathrm{SD}$ & Median & Range & Mean & SD & Median & Range \\
\hline Speech intelligibility & 6 & - & 6 & - & 12.09 & 2.74 & 13 & $7-16$ & 12.94 & 2.04 & 13 & $8-16$ \\
\hline Ease of communication & 4 & - & 4 & - & 8.09 & 2.39 & 9 & $5-12$ & 8.71 & 2.15 & 9 & $2-14$ \\
\hline Efficiency of noise reduction & 3 & - & 3 & - & 4.55 & 0.69 & 5 & $3-5$ & 3.83 & 1.27 & 4 & $0-5$ \\
\hline Naturalness of speech & 1 & - & 1 & - & 2 & 0.78 & 2 & $1-3$ & 2.06 & 0.42 & 2 & $1-3$ \\
\hline Music perception & 2 & - & 2 & - & 2.73 & 0.47 & 3 & $2-3$ & 2.53 & 0.56 & 3 & $1-3$ \\
\hline
\end{tabular}

Table 6 Mean, standard deviation (SD), median and range of scores obtained by premium device users across domains based on daily usage duration

\begin{tabular}{|c|c|c|c|c|c|c|c|c|c|c|c|c|}
\hline \multirow[t]{2}{*}{ Daily usage duration } & \multicolumn{4}{|c|}{ Less than $5 \mathrm{~h}(n=5)$} & \multicolumn{4}{|c|}{$5-10 \mathrm{~h}(n=8)$} & \multicolumn{4}{|c|}{ More than $10 \mathrm{~h}(n=42)$} \\
\hline & Mean & SD & Median & Range & Mean & SD & Median & Range & Mean & SD & Median & Range \\
\hline Speech intelligibility & 12 & 1.16 & 12 & $11-13$ & 12 & 2.07 & 12.5 & $9-14$ & 13.55 & 2.61 & 14 & $6-17$ \\
\hline Ease of communication & 9.75 & 1.89 & 10.5 & $7-11$ & 7.63 & 2.39 & 6 & $6-12$ & 9.88 & 2.41 & 10 & $4-14$ \\
\hline Efficiency of noise reduction & 3 & 0.82 & 3 & $2-4$ & 3.38 & 1.41 & 3.5 & $1-5$ & 4.05 & 1.08 & 4 & $1-5$ \\
\hline Naturalness of speech & 1.5 & 0.58 & 1.5 & $1-2$ & 1.88 & 0.35 & 2 & $1-2$ & 2.17 & 0.49 & 2 & $1-3$ \\
\hline Music perception & 2.75 & 0.96 & 2.5 & $2-4$ & 2.38 & 0.92 & 3 & $1-3$ & 2.78 & 0.57 & 3 & $1-4$ \\
\hline
\end{tabular}


and volume settings resulting in better directional hearing. These findings agree with the claims of most manufacturers regarding the outcomes of mid-range and premium models compared to basic hearing aids [5, 24]. However, the results from the rest of the listening domains are questionable based on the manufacturer's claim. Among the participants, about fifty percent have undergone hearing aid fitting after a period of 3-5 years post-onset of hearing loss. The same trend has been reported in various literature $[1,2]$. It could be possibly due to the psychological conflicts in accepting the hearing loss and cosmetic concerns towards the hearing aid fitting.

Rated performance of different categories of the hearing aids in the monaural and binaural conditions revealed no significant differences across any of the domains assessed. Similar findings were reported by Walden et al. [25]. They compared the performance of linear hearing aids with Wide Dynamic Range Compression (WDRC) devices using Profile of Hearing aid Benefit (PHAB) and revealed minimal differences in speech perception scores across the devices. Similarly, Wu et al. [8] compared the performance of premium hearing aids with that of basic hearing aids. The measurements were obtained in laboratory conditions objectively and also based on questionnaire responses from subjects. Objective test results showed better results with premium hearing aids over basic hearing aids. However, subjective outcome reports were not strong enough to claim the benefit of premium devices over basic devices. The study also highlighted that older adults are less likely to benefit from the features specific to premium hearing aids than younger age groups. It may also depend on lifestyle and socio-economic background (Fig. 3).

The long-term duration of hearing aid showed a slight improvement in mean scores of speech intelligibility and ease of communication irrespective of the hearing aid category. Neuroplastic changes caused due to the amplification and getting acclimatized with hearing aids over time may be the contributing factors for this improvement [26]. However, the overall effect of duration of usage of hearing aids (in years) in all other domains was not significant. Regular and continuous use of hearing aids are recommended for better benefit with hearing aids for individuals with hearing loss [25]. This recommendation would have been made considering the neural plasticity phenomenon utilized with acoustic stimulation and adaptation to the hearing aid processed speech. However, in the comparison of daily usage durations, there were no significant differences across listening domains in any of the hearing aid categories but on observation there is a slight improvement in the rated scores about the speech intelligibility and ease of communication with the duration of hearing aid usage.

The cost effectiveness of the device was assessed among two different categories of hearing aid users. This domain was assessed assuming that if the advanced features worked efficiently in real life and those who procured it would have been satisfied better. On the other hand, those patients using the basic category of hearing aids owing to less features would have experienced much more listening difficulties and they would have rated the cost effectiveness negatively. Contrary to the above assumption, the overall cost effectiveness was reported to be better by the entry-level hearing aid users than the users of premium models.

It is important to note that among the participants, those who had financial constraints to procure premium category devices, trials were given with different models of basic category devices only. A blinded trial with both basic and premium category hearing aids for every client would enhance the reliability of the perceptual rating of hearing aid performance.
Fig. 3 Mean scores obtained across different listening domains corresponding to the duration of hearing aid usage (in years)

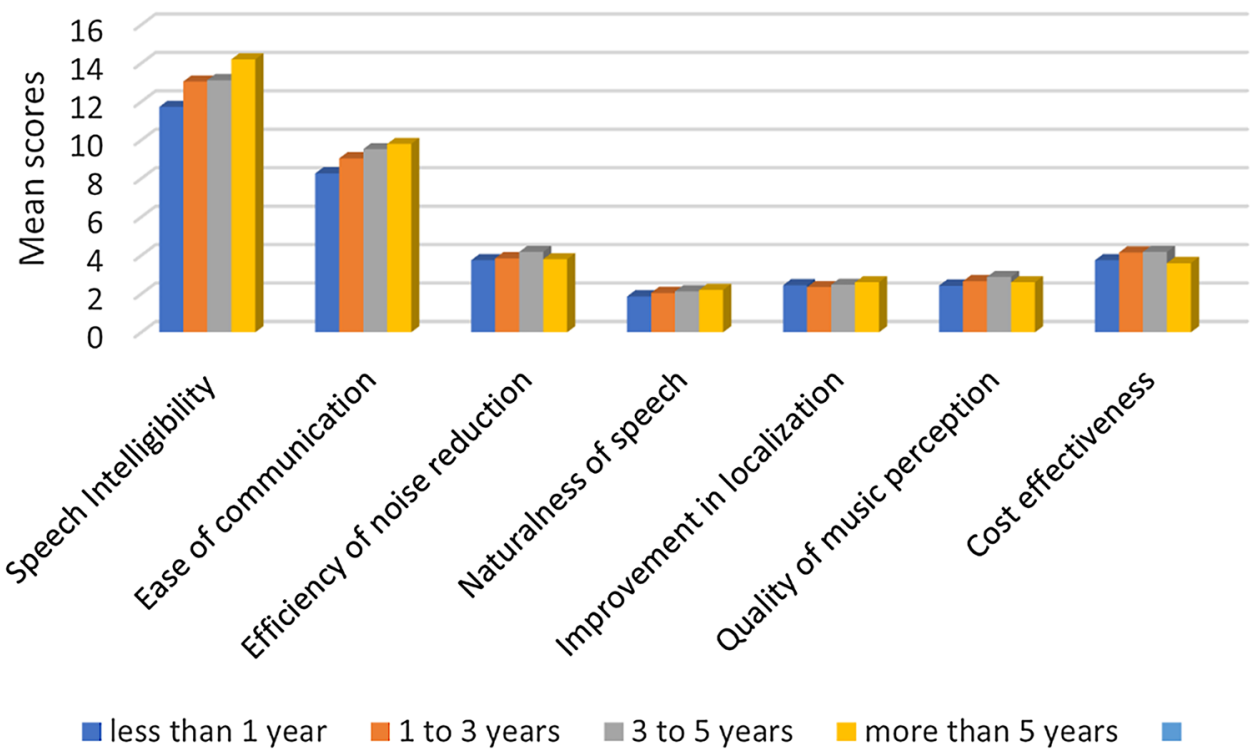


The reduced rating on cost effectiveness by users of premium hearing aids can be due to the higher expectation imposed by the higher cost of hearing aids. Moreover, the users of premium hearing aids may expect quality near to natural hearing and more comfort in using the hearing aids than those with basic hearing aids. In the current study, it is difficult to differentiate whether the reduced cost effectiveness rated by users of premium category of the hearing aid is due to the lack of performance of the hearing aids in challenging listening environment, the higher cost of the device or the higher expectation imposed by the cost. Lifestyle could be considered as another potential factor that affects cost effectiveness. If the social and professional life of a client extensively utilizes the advanced features that are present in the premium models in his/her daily communication, those individuals are more likely to report better cost effectiveness for premium hearing aids. While selecting a premium category of hearing aids for a patient who can afford it, other than the audiometric configuration, the listening requirements needed to be taken care of. A realistic explanation regarding the benefit of hearing aids may also improve the appropriate selection and satisfaction with hearing aids. Furthermore, routine audiological evaluations and reprogramming of devices as per the requirement and listening issues reported by the clients could also improve the satisfaction with the hearing aids.

The current study reported that almost all the premium category users were not using wireless connectivity features of their devices. Precise explanations regarding the features and controls of the device by audiologists during the initial hearing aid trial session itself would definitely help the client foresee whether those advanced features match with his/her listening needs.

\section{Limitations and future directions}

The study was performed by conducting comparisons across persons using different categories of hearing aid. Those users of basic category devices who reported financial constraints during the hearing aid trial were not assessed their performance with the premium category devices. Hence, a prospective study design with controlled variables would be required for further confirming the findings of the present study. The current results could be validated by conducting a comparison study by providing both the categories of hearing aids to participants to get an unbiased rating of these hearing aids in a blindfolded way. Moreover, categorization of different hearing aids in terms of their lifestyle was not done and the educational backgrounds were not considered.

\section{The implication of the study}

Apart from the conventional fitting methods, successful hearing aid fitting can be achieved if the client is satisfied in the daily listening situations and the incorporation of technology to sense the environment automatically. Among the non-audiological factors, the cost of the devices and expectations from the hearing aids are the key elements that can affect satisfaction with hearing aid usage. For patients willing to procure premium hearing aids, the extent of benefits needs to be explained. Moreover, trials with both basic and premium model of hearing aids need to be given to get a perceptual score from both the devices on major listening domains like speech discrimination in quiet and noise, the naturalness of speech, noise reduction efficiency and localization. Furthermore, based on the ratings on overall cost effectiveness across the listening domains, the audiologist may recommend the appropriate hearing devices. This would enhance the hearing aid outcome and the satisfaction from hearing aid usage.

\section{Conclusion}

The current study compared the benefit of basic hearing aids with mid-range or premium models across different manufacturers. Due to the automatic features like adaptive volume and program adjustments, ease of communication in daily life was reported to be comparatively better by users of premium hearing aid users. There were no notable differences between basic and premium category devices in other listening domains assessed. Cost effectiveness was reported to be better by the users of basic hearing aids. A prospective and controlled paired series comparison of hearing aid performance across different categories of hearing aids needs to be performed in future studies to reconfirm these findings.

Supplementary Information The online version contains supplementary material available at https://doi.org/10.1007/s00405-021-07240-3.

Acknowledgements The authors would like to express gratitude to the Director, All India Institute of Speech and Hearing (AIISH), Mysore, affiliated to the University of Mysore. The authors express their sincere gratitude to all the participants for their support and co-operation.

Author contributions PP was involved in study design, stimulus preparation, data collection, analysis of the data, interpretation and writing the manuscript; AS was involved in study design, stimulus preparation, data collection, analysis of the data, interpretation and writing the manuscript; JB was involved in study design, stimulus preparation, data collection, analysis of the data, interpretation and writing the manuscript; AV was involved in study design, stimulus preparation, data collection, analysis of the data, interpretation and writing the manuscript.

Funding None. 
Availability of data and materials (data transparency) Yes.

Code availability Not applicable.

\section{Declarations}

Conflict of interest The authors declare that they have no conflict of interest.

Ethics approval AIISH ethical committees approved the study method for bio-behavioral research.

Consent to participate Written informed consent taken prior commencing the data collection.

Consent for publication Yes, informed content was obtained from subjects for participating in the study.

\section{References}

1. Chisolm TH, Johnson CE, Danhauer JL, Portz LJ, Abrams HB, Lesner S, Newman CW (2007) A systematic review of healthrelated quality of life and hearing aids: final report of the American Academy of Audiology Task Force on the Health-Related Quality of Life Benefits of Amplification in Adults. J Am Acad Audiol 18(2):151-183

2. National Institute on Deafness and Other Communication Disorders. Hearing loss and older adults. 2016. Available at: https:// www.nidcd.nih.gov/health/hearing-loss-older-adults

3. Blazer DG, Domnitz S, Liverman CT (2016) Hearing health care for adults: priorities for improving access and affordability.

4. Kochkin S (2009) MarkeTrak VIII: 25-year trends in the hearing health market. Hear Rev 16(11):12-31

5. Know, H. A. (2020). Review of the ReSound ONE Hearing Aid.

6. Jorgensen EJ, Stangl E, Chipara O, Hernandez H, Oleson J, Wu YH (2020) GPS predicts stability of listening environment characteristics in one location over time among older hearing aid users. Int J Audiol 60:1-13

7. Yund EW, Buckles KM (1995) Multichannel compression hearing aids: Effect of number of channels on speech discrimination in noise. J Acoust Soc Am 97(2):1206-1223

8. Wu YH, Stangl E, Chipara O, Hasan SS, DeVries S, Oleson J (2019) Efficacy and effectiveness of advanced hearing aid directional and noise reduction technologies for older adults with mild to moderate hearing loss. Ear Hear 40(4):805-822. https://doi.org/ 10.1097/AUD.0000000000000672

9. Keidser G, O’Brien A, Hain JU, McLelland M, Yeend I (2009) The effect of frequency-dependent microphone directionality on horizontal localization performance in hearing-aid users. Int $\mathbf{J}$ Audiol 48(11):789-803

10. Munro KJ, Puri R, Bird J, Smith M (2016) Using probe-microphone measurements to improve the match to target gain and frequency response slope, as a function of earmould style, frequency, and input level. Int J Audiol 55(4):215-223
11. Aazh H, Moore BC (2007) The value of routine real ear measurement of the gain of digital hearing aids. J Am Acad Audiol 18(8):653-664

12. Valente M (2007) Guideline for audiologic management of the adult patient. Audiology Online

13. Robinson K, Gatehouse S, Browning GG (1996) Measuring patient benefit from otorhinolaryngological surgery and therapy. Ann Otol Rhinol Laryngol 105(6):415-422

14. Dillon H, James A, Ginis J (1997) Client oriented scale of improvement (COSI) and its relationship to several other measures of benefit and satisfaction provided by hearing aids. J Am Acad Audiol 8:27-43

15. Cox RM, Alexander GC (1995) The abbreviated profile of hearing aid benefit. Ear Hear 16(2):176-186

16. Cox RM, Alexander GC (2002) The International Outcome Inventory for Hearing Aids (IOI-HA): psychometric properties of the English version: El Inventario International de Resultados para Auxiliares Auditivos (IOI-HA): propiedades psicometricas de la version en ingles. Int J Audiol 41(1):30-35

17. Walden BE, Demorest ME, Hepler EL (1984) Self-report approach to assessing benefit derived from amplification. J Speech Lang Hear Res 27(1):49-56

18. Bertoli S, Bodmer D, Probst R (2010) Survey on hearing aid outcome in Switzerland: associations with type of fitting (bilateral/ unilateral), level of hearing aid signal processing, and hearing loss. Int J Audiol 49(5):333-346

19. Bertoli S, Staehelin K, Zemp E, Schindler C, Bodmer D, Probst R (2009) Survey on hearing aid use and satisfaction in Switzerland and their determinants. Int J Audiol 48(4):183-195

20. Easwar V, Boothalingam S, Chundu S, Manchaiah VK, Ismail SM (2013) Audiological practice in India: an internet-based survey of audiologists. Indian J Otolaryngol Head Neck Surg 65(3):636-644

21. Kumar S, Maheshwari V, Prabhu J, Prasanna M, Jayalakshmi P, Suganya P, Malar BA, Jothikumar R (2020) Social economic impact of COVID-19 outbreak in India. Int J Pervas Comput Commun

22. Caspary DM, Raza A, Armour BL, Pippin J, Arneric SP (1990) Immunocytochemical and neurochemical evidence for age-related loss of GABA in the inferior colliculus: implications for neural presbycusis. J Neurosci 10(7):2363-2372

23. Frey, F. (2017). SPSS (Software). In: The International Encyclopedia of Communication Research Methods, 1-2.

24. Palmer, C. A. T. H. E. R. I. N. E. (2019). Hearing Aids 2019: Today's Technology.

25. Walden BE, Surr RK, Cord MT, Edwards B, Olson L (2000) Comparison of benefits provided by different hearing aid technologies. J Am Acad Audiol 11(10):540-560

26. Glick H, Sharma A (2021) The brain on hearing aids: can treatment with hearing aids improve neurocognitive function in agerelated hearing loss? Hear Rev 28(1):28-32

Publisher's Note Springer Nature remains neutral with regard to jurisdictional claims in published maps and institutional affiliations. 